\title{
Hydrogenated Ge Nanocrystals: Band Gap Evolution with Increasing Size
}

\author{
P. Alfaro, A. Miranda, A.E. Ramos, and M. Cruz-Irisson \\ Sección de Estudios de Posgrado e Investigación, ESIME-Culhuacan, \\ Instituto Politécnico Nacional, Av. Santa Ana 1000, Código Postal 04430, México, D.F., México
}

Received on 4 April, 2005

\begin{abstract}
The electronic band structure of various Ge quantum wires of different sizes, with hydrogenated surfaces, is studied using a nearest-neighbor empirical tight-binding Hamiltonian by means of a sp $\mathrm{s}^{*}$ atomic orbitals basis set. We suppose that the nanostructures have the same lattice structure and the same interatomic distance as in bulk Ge and that all the dangling bonds are saturated with hydrogen atoms. These atoms are used to simulate the bonds at the surface of the wire and sweep surface states out of the fundamental gaps. One of the most important features is a clear broadening of the band gap due the quantum confinement. Comparing to experimental data, we conclude that, similar to the case of Si, the size dependent PL in the near infrared may involve a trap in the gap of the nanocrystals.
\end{abstract}

Keywords: Electronic band structure; Ge quantum wires; Empirical tight-binding

\section{INTRODUCTION}

During the last years photoluminescence properties of nanometer materials have motivated a great deal of experimental and theoretical research effort because they exhibit favorable applications in opto-electronic devices. The quantum confinement effect of photoexcited carriers within nanocrystallites was mainly proposed to be responsible for the visible luminescence from these materials.

In the present work, we employ a semiempirical tightbinding model (STBM) to study the electronic structure of hydrogenated Ge nanowires that, by construction, reproduces the correct band gap of bulk $\mathrm{Ge}$ in the limit of infinite supercell size. This feature allows us to overcome a difficulty common to some other models: when the supercell sizes becomes very large and the calculations become computationally almost intractable, some models cannot be relied upon. Furthermore, the simplicity of an STBM makes calculations for very large systems [1,2].

Recently, attempts have been made to fabricate quantum size structures based on silicon or germanium, in which usually indirect optical transition take place between the band gap so that the radiative efficiency is limited to a very small value. The bulk Ge has a larger dielectric constant and smaller carrier masses compared to the bulk Si. Moreover, in Ge, the gap $\operatorname{direct}\left(E_{0} \approx 0.9 \mathrm{eV}\right)$ is close to the indirect gap $\left(E_{g} \approx 0.76 \mathrm{eV}\right)$. Then, it is considered that quantum confinement effects would appear more pronounced in $\mathrm{Ge}$ than in $\mathrm{Si}$, and $\mathrm{Ge}$ nanocrystals would exhibit a direct- gap semiconductor nature [3]. Takeoka and coworkers [4] have observed a size dependent photoluminescence (PL) from nanostructures of indirect-gap in the nearinfrared region which is closer to the band gap of bulk Ge and which seems more compatible with the quantum confinement model. On the other hand, the importance of the localized levels at the surface of nanostructures in the PL was pointed out by several authors [5,6]. Because the mechanism of PL from nanostructures of indirect gap materials is controversial and not well understood, it is important to clarify theoretically the intrinsic electronic properties of these quantum-confined structures. The comparison of the theoretical results with ex- perimental data will shed a new light on the mechanism of PL. Thus, the purpose of this work is to provide a theoretical description of the electronic properties of Ge nanocrystals and to compare it with experimental results.

In the bulk crystal of $\mathrm{Ge}$, the electron-hole recombination is possible only through phonon emission or absorption because the wave vector difference between the conduction band bottom and the valence band top must be compensated. One of the most effective means to convert an indirect optical transition into a direct one is to form a supercell structure by which the size of the Brillouin zone is reduced and the conduction band bottom is folded onto the $\Gamma$ point, resulting in a direct-gap material [7]. On the other hand, in nanostructures such as an isolated quantum dot, the periodicity due to a superstructure is absent and the above picture of zone folding is not applicable straightforwardly. In these structures, the electronic states become completely discrete as in atoms and molecules and the optical matrix element between a pair of discrete states must be evaluated to identify whether that transition is optically allowed or not. This is the most legitimate picture. However, in a relatively large nanostructure, we can employ approximately the picture of zone folding. If the envelope function of carriers confined in a nanostructure have a sizable Fourier component at the wave vector corresponding to the indirect-gap transition, that Fourier component plays the same role as phonons in the bulk material and the direct optical transitions becomes allowed.

In the next section, we describe the supercell structure and the Hamiltonian used in our model and calculation scheme. In Sec. III the numerical results are presented and compared with experimental data. Finally, the main conclusions of our study are summarized in Sec. IV.

\section{MODEL AND CALCULATION SCHEME}

We consider free standing, infinitely long and homogeneous quantum wires of rectangular cross section with the wire axis along the [001] direction, and wire surfaces which correspond to (110) surfaces of bulk Ge. Each surface dan- 
gling bond is saturated with a hydrogen atom. The system has translational symmetry in the $\mathrm{z}$ axis with period equal a lattice constant $a$.The structure of the narrowest wire considered is illustrated as show in the Fig. 1(a). This wire has eight $\mathrm{Ge}$ atoms per unit cell (8-atom supercell) which, when viewed along the wire axis, form a square array [Fig. 1(b)].

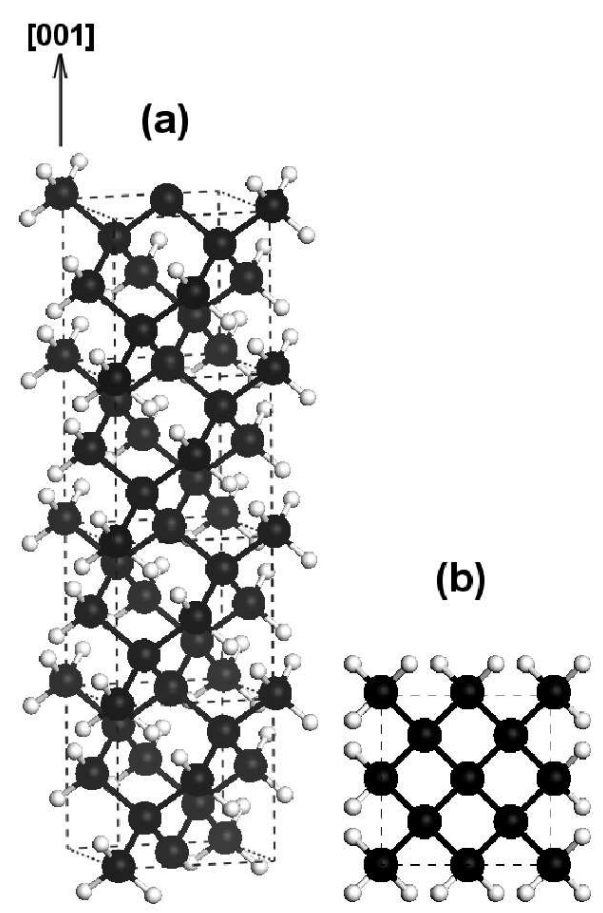

FIG. 1: (a) Hydrogen-passivated crystalline Ge nanowire along the [001] direction, and (b) cross-sectional viewed from the top.

A 32-atom supercell is built by joining four such cubes in the $x-y$ plane, leading to a structure with parameters $a_{x}=$ $a_{y}=2 a$ and $a_{z}=a$, the width of this nanowire is $d=1.132$ nm.

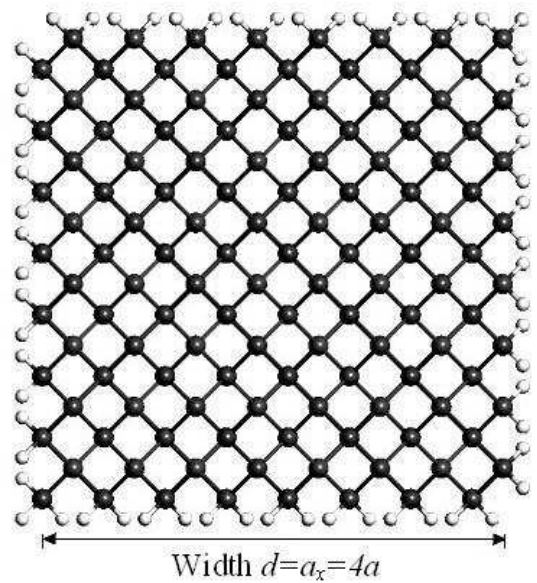

FIG. 2: Cross-sectional view of 128-atom supercell, the dangling bonds of the surface are saturated with $\mathrm{H}$.
For a 128-atom supercell, the parameters are $a_{x}=a_{y}=4 a$ and $a_{z}=a$, with translational symmetry only in de z-axis; an example of such supercell is shown in Fig. 2.

We suppose that the nanostructures have the same lattice structure and the same interatomic distance than bulk Ge and that all the dangling bonds are saturated with hydrogen atoms described by $1 s$ orbital. For simplicity we suppose also that there is no hydrogen-hydrogen interaction. These atoms are used to simulate the bonds at the surface of the wire and sweep surface states out of the fundamental gaps [8]. We assume that the $\mathrm{H}$-saturated dangling bonds on the surface of the wire have the natural $\mathrm{H}-\mathrm{Ge}$ bond length. We are aware that we are simplifying enormously the surface description, ignoring other possible saturators and surface reconstruction [9-11]. Clearly, to study PL phenomena, this description is insufficient.

Since the experimental data are in fact the average value of physical quantities from many equivalent-size nanostructures of different atomic configurations, the calculational accuracy for a particular atomic configuration is rather unimportant. The important point is to study numerous different nanostructures, and study the tendency of their behaviors as a whole. In this respect, it is best to use the TB method, which is far more economical both in time and money than, say, first-principle electronic calculation, in accordingly allows us to treat a large number of different nanostructures.

As we are interested in describing the band-structure modifications around the fundamental gap, the minimal basis capable of describing it is the $s p^{3} s *$ basis. We have used the parameters of Vogl, Hjalmarson, and Dow [12], which reproduce a $0.76 \mathrm{eV}$ indirect-gap in bulk crystalline Ge. There is also a $s p^{3} s *$ TB calculation scheme of the optical absorption spectrum of Ge nanocrystals but the authors did not pay attention to the gap energy $[13,14]$.

The on-site energy of the $\mathrm{H}$ and the $\mathrm{Ge}-\mathrm{H}$ orbital interaction parameters are taken as $E_{H}=0.205 \mathrm{eV}, s s \sigma_{G e-H}=-3.618 \mathrm{eV}$, and $s p \sigma_{G e-H}=4.081 \mathrm{eV}$ respectively, which are obtained by fitting the energy levels of $\mathrm{GeH}_{4}$ calculated in Local Density Approximation [15].

We solve for the electronic states by diagonalizing the TB Hamiltonian directly. The dimension of the TB Hamilonian matrix is $5 \times N_{G e}+N_{H}$, where $\mathrm{N}_{G e}$ and $\mathrm{N}_{H}$ are numbers of $\mathrm{Ge}$ and $\mathrm{H}$ atoms in the nanowire supercell. We are calculated quantum-wires band structure for seven wire widths $(\sim 0.5-4$ $\mathrm{nm})$ in a systematic manner.

\section{RESULTS}

The results for the band gap evolution for hydrogenated germanium nanocrystals with respect to the width size $(d)$ are plotted in Fig. 3. The calculated lowest unoccupied conduction band state energy and highest occupied valence band state energy are shown.

Note the quantum size effect: as the width of the wire decrease, the highest occupied state decrease in energy and the lowest unoccupied state increases.

For the largest nanowire (with width $d=3.97 \mathrm{~nm}$ ), the lowest unoccupied state is very close to the bottom of the bulk 


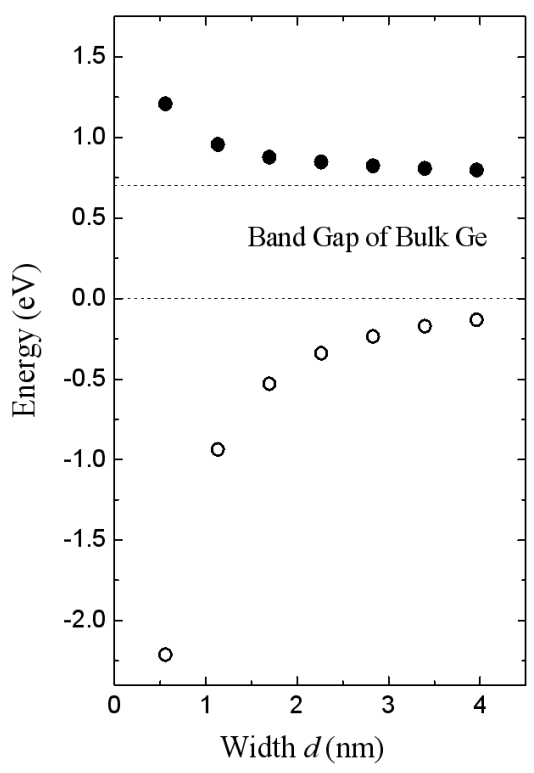

FIG. 3: TB band gap evolution of Ge wires. (a) The lowest unoccupied conduction state $(\bullet)$ and the highest occupied valence state $(\bigcirc)$ as a function of the width $d$. The band gap of bulk Ge is indicated by the dash lines.

conduction band $(0.79$ vs. $0.76 \mathrm{eV})$, and the highest occupied state is very near to the top of the valence band (-0.133 vs. 0$)$. The horizontal dash lines are the bulk band edges. Furthermore, the band borders shift asymmetrically. That the valence band shifts more rapidly is consistent with the difference in the band curvatures.

Figure 4 indicates the comparison the our calculations with the experimental data [4]. These results show a size dependent PL in the near-infrared region which is closer to the band gap of bulk Ge and which seems is compatible with the quantum confinement model.

The observed size dependence the PL spectrum shown in Fig. 4 is very similar to those of $\mathrm{Si}$ and other semiconductor nanocrystals. This strongly suggest that the PL peak observed originates from the recombination of electron-hole pairs between the widened band gap of Ge nanocrystals. The near infra-red PL of Ref [4] has a clear dependence on size but is much lower than our theoretical calculations even if we consider the broadening of the experimental spectra $(0.4-1.0 \mathrm{eV})$ and the uncertainty on the crystallites sizes below $3 \mathrm{~nm}$.

The variation of the PL energy with size can be explained if the PL is related to the recombination of an electron (hole) trapped on a defect with the free hole (electron) in the crystal- lite.

\section{CONCLUSION}

In this work, we have show that the electronic structure of surface-hydrogenated Ge nanocrystals, from small to large supercells with bulk properties, can be calculated with a single

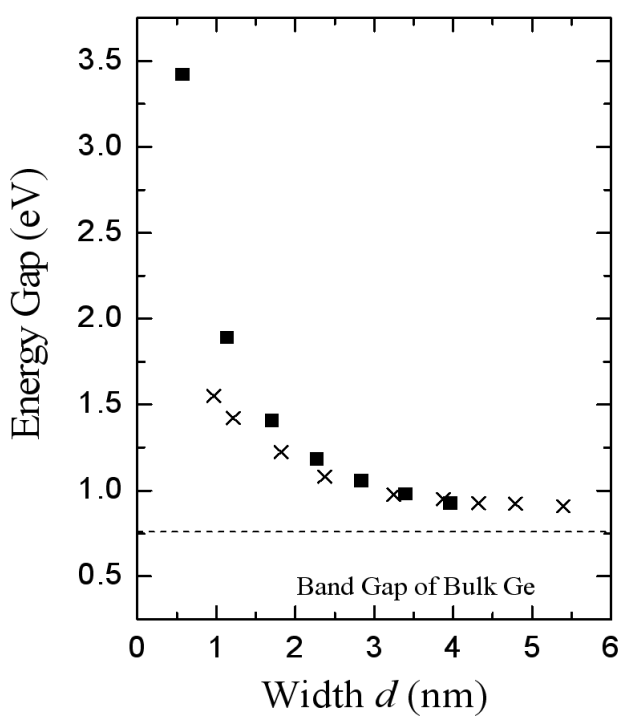

FIG. 4: Comparison of the experimental PL peak (x) for Ge nanocrystals with our calculations $(\mathbf{\square})$. The experimental data are presented by digitizing the plot of Ref. [4].

TB model. We have studied the band gap evolution of hydrogenated Ge nanocrystals as a function of the size. After the compare our theoretical calculations with experimental data we can conclude that, the broadening of the band gap is due the quantum confinement and similar to the case of $\mathrm{Si}$, the size dependent PL in the near infrared may be involves a trap in the gap of the nanocrystals. The supercell model can be further improved to include other saturators of the surface (e.g. oxygen), surface and structural relaxation and amorphization.

\section{Acknowledgement}

This work has been partially supported by Project No. CGPI-IPN 20040062. The use of the computing facilities of DGSCA-UNAM is acknowledged.
[1] Tight-Binding Approach to Computational Materials Science, edited by P.E.A. Turchi, A. Gonis, and L. Colombo, MRS Symposia Proceedings 491, Materials Research Society, Warrendale, PA, 1998.
[2] H. Yorikawa, T. Sato, and S. Muramatsu, Phys. Rev. B 95, 3569 (2004).

[3] J.R. Heath, J.J. Shiang, and A.P. Alivisatos, J. Chem. Phys. 101, 1607 (1994). 
[4] S. Takeoka, M. Fujii, S. Hayashi, and K. Yamamoto, Phys. Rev. B 58, 7921 (1998).

[5] M.V. Wolkin et al., Phys. Rev. Lett. 82, 197 (1999).

[6] S. Louhibi, N. Sekkal, N. Benkhettou, and N.E. Chabane-Sari, Superlattices and Microstructures 37, 115 (2005).

[7] S.S. Iyer and Y.H. Xie, Science 260, 40 (1993).

[8] D. Guzmán, U.Corona, and M. Cruz, J. Lumin. 102, 487 (2003).

[9] J. Koga et al., J. Non-Cryst. Solids 293-295, 630 (2001).

[10] J. Koga, K. Nishio, F. Yonezawa, and T. Yamaguchi, Phys. E 15, 182 (2002).
[11] H.-Ch. Weissker, J. Furthmüller, and F. Bechstedt, Surf. Sci. 566-568, 961 (2004)

[12] P. Vogl, H.P. Hjalmarson, and J.D. Dow, J. Phys. Chem. Solids 44, 365 (1983).

[13] M. Palummo, G. Onida, and R. Del Sole, Phys. Stat. Sol. (a) 175, 23 (1999).

[14] M. Palummo et al., Phys. Stat. Sol. (b) 224, 247 (2001).

[15] DMOL User Guide, Molecular Simulation, San Diego, 1996. 